\title{
A NEW PROOF OF EXISTENCE OF SOLUTIONS FOR FOCUSING AND DEFOCUSING GROSS-PITAEVSKII HIERARCHIES
}

\author{
THOMAS CHEN AND NATAŠA PAVLOVIĆ \\ (Communicated by Hart F. Smith)
}

\begin{abstract}
We consider the cubic and quintic Gross-Pitaevskii (GP) hierarchies in $d \geq 1$ dimensions for focusing and defocusing interactions. We present a new proof of existence of solutions that does not require the a priori bound on the spacetime norm, which was introduced in the work of Klainerman and Machedon and used in our earlier work.
\end{abstract}

\section{INTRODUCTION}

In this paper, we investigate the existence of solutions to the Gross-Pitaevskii (GP) hierarchy, with focusing and defocusing interactions. We present a new proof of existence of solutions that does not require the a priori bound on the spacetime norm, which was introduced in the work of Klainerman and Machedon [19], and which we used in our earlier work [6].

The GP hierarchy is a system of infinitely many coupled linear PDEs describing a Bose gas of infinitely many particles, interacting via delta interactions. Some GP hierarchies (defocusing energy subcritical and focusing $L^{2}$-subcritical) can be obtained as limits of BBGKY hierarchies of $N$-particle Schrödinger systems of identical bosons, in the limit $N \rightarrow \infty$. In the recent literature on this topic, there is a particular interest in the special class of factorized solutions to GP hierarchies, which are parametrized by solutions of a nonlinear Schrödinger (NLS) equation. In this context, the NLS is interpreted as the mean field limit of an infinite system of interacting bosons in the so-called Gross-Pitaevskii limit. We refer to [10, 11, 12, 20, 19, 24 and the references therein, and also to [1, 3, 5, 9, 13, 14, 15, 17, 16, 18, 26]. For recent mathematical developments focusing on the related problem of BoseEinstein condensation, we refer to [2, 21, 22, 23] and the references therein.

In a landmark series of works, Erdös, Schlein, and Yau 10, 11, 12 provided the derivation of the cubic NLS as a dynamical mean field limit of an interacting Bose gas for a very general class of systems. The construction requires two main steps:

(i) Derivation of the GP hierarchy as the $N \rightarrow \infty$ limit of the BBGKY hierarchy of density matrices associated to an $N$-body Schrödinger equation. The latter is defined for a scaling where the particle interaction potential tends to a delta distribution, and where total kinetic and total interaction energy have the same order of magnitude in powers of $N$.

Received by the editors December 22, 2010 and, in revised form, June 17, 2011. 2010 Mathematics Subject Classification. Primary 35Q55. 
(ii) Proof of the uniqueness of solutions for the GP hierarchy. It is subsequently verified that for factorized initial data, the solutions of the GP hierarchy are determined by a cubic NLS for systems with 2-body interactions.

The proof of the uniqueness of solutions of the GP hierarchy is the most difficult part of this program, and it is obtained in 10, 11, 12] by use of highly sophisticated Feynman graph expansion methods inspired by quantum field theory.

In [19, Klainerman and Machedon presented a different method to prove the uniqueness of solutions for the cubic GP hierarchy in $d=3$, in a different space of solutions than in 10, 11. Their approach uses Strichartz-type spacetime bounds on marginal density matrices, and a sophisticated combinatorial result, obtained via a certain "boardgame argument" (which is a reformulation of a method developed in [10, 11]). The analysis of Klainerman and Machedon requires the assumption of an a priori spacetime bound which is not proven in [19]. In [20, Kirkpatrick, Schlein, and Staffilani proved that this a priori spacetime bound is satisfied, locally in time, for the cubic GP hierarchy in $d=2$, by exploiting the conservation of energy in the BBGKY hierarchy, in the limit as $N \rightarrow \infty$. In [5], we proved that the analogous a priori spacetime bound holds for the quintic GP hierarchy in $d=1,2$.

In [6], we prove the existence and uniqueness of solutions in the spaces used by Klainerman and Machedon in [19], and provide an estimate that gives a precise meaning to their a priori assumption. For the proof, we introduce a natural topology on the space of sequences of $k$-particle marginal density matrices

$$
\mathfrak{G}=\left\{\Gamma=\left(\gamma^{(k)}\left(x_{1}, \ldots, x_{k} ; x_{1}^{\prime}, \ldots, x_{k}^{\prime}\right)\right)_{k \in \mathbb{N}} \mid \operatorname{Tr} \gamma^{(k)}<\infty\right\}
$$

and invoke a contraction mapping argument. Accordingly, we prove in [6] local well-posedness for the cubic and quintic GP hierarchies, in various dimensions.

In [6], we use the Klainerman-Machedon a priori assumption on the boundedness of a certain spacetime norm for both the uniqueness and the existence parts of the proof (which are obtained in the same step, via the contraction mapping argument).

In this paper, we give a new proof of the existence of solutions for focusing and defocusing $p$-GP hierarchies, without assuming any a priori spacetime bounds. However, we prove as an a posteriori result that the Klainerman-Machedon spacetime bound is indeed satisfied by this solution.

Organization of the paper. In Section 2 we introduce the GP hierarchy and the spaces that we use to analyze the hierarchy. In Section 3 we state the main result of this paper, Theorem 3.1, and we present a proof of this theorem. The proof uses a free Strichartz estimate, as well as an iterated version of the Strichartz estimate, both of which are presented in Appendix A. The existence of solutions of $p$-GP hierarchies with truncated initial data is addressed in Appendix B.

\section{THE MODEL}

In this section, we introduce the mathematical model analyzed in this paper. We will mostly adopt the notation and definitions from [6], and we refer to [6] for motivations and more details.

2.1. The spaces. We introduce the space

$$
\mathfrak{G}:=\bigoplus_{k=1}^{\infty} L^{2}\left(\mathbb{R}^{d k} \times \mathbb{R}^{d k}\right)
$$


of sequences of density matrices

$$
\Gamma:=\left(\gamma^{(k)}\right)_{k \in \mathbb{N}},
$$

where $\gamma^{(k)} \geq 0, \operatorname{Tr} \gamma^{(k)}=1$, and where every $\gamma^{(k)}\left(\underline{x}_{k}, \underline{x}_{k}^{\prime}\right)$ is symmetric in all components of $\underline{x}_{k}$, and in all components of $\underline{x}_{k}^{\prime}$, respectively, i.e.

$$
\gamma^{(k)}\left(x_{\pi(1)}, \ldots, x_{\pi(k)} ; x_{\pi^{\prime}(1)}^{\prime}, \ldots, x_{\pi^{\prime}(k)}^{\prime}\right)=\gamma^{(k)}\left(x_{1}, \ldots, x_{k} ; x_{1}^{\prime}, \ldots, x_{k}^{\prime}\right)
$$

holds for all $\pi, \pi^{\prime} \in S_{k}$.

For brevity, we will denote the vector $\left(x_{1}, \cdots, x_{k}\right)$ by $\underline{x}_{k}$ and similarly the vector $\left(x_{1}^{\prime}, \cdots, x_{k}^{\prime}\right)$ by $\underline{x}_{k}^{\prime}$.

The $k$-particle marginals are assumed to be Hermitian,

$$
\gamma^{(k)}\left(\underline{x}_{k} ; \underline{x}_{k}^{\prime}\right)=\overline{\gamma^{(k)}\left(\underline{x}_{k}^{\prime} ; \underline{x}_{k}\right)} \text {. }
$$

We call $\Gamma=\left(\gamma^{(k)}\right)_{k \in \mathbb{N}}$ admissible if $\gamma^{(k)}=\operatorname{Tr}_{k+1} \gamma^{(k+1)}$, that is,

$$
\gamma^{(k)}\left(\underline{x}_{k} ; \underline{x}_{k}^{\prime}\right)=\int d x_{k+1} \gamma^{(k+1)}\left(\underline{x}_{k}, x_{k+1} ; \underline{x}_{k}^{\prime}, x_{k+1}\right)
$$

for all $k \in \mathbb{N}$.

Let $0<\xi<1$. We define

$$
\mathcal{H}_{\xi}^{\alpha}:=\left\{\Gamma \in \mathfrak{G} \mid\|\Gamma\|_{\mathcal{H}_{\xi}^{\alpha}}<\infty\right\}
$$

where

$$
\|\Gamma\|_{\mathcal{H}_{\xi}^{\alpha}}=\sum_{k=1}^{\infty} \xi^{k}\left\|\gamma^{(k)}\right\|_{H_{k}^{\alpha}\left(\mathbb{R}^{d k} \times \mathbb{R}^{d k}\right)},
$$

with

$$
\left\|\gamma^{(k)}\right\|_{H_{k}^{\alpha}}=\left(\int d \underline{x}_{k} d \underline{x}_{k}^{\prime}\left|S^{(k, \alpha)} \gamma^{(k)}\left(\underline{x}_{k} ; \underline{x}_{k}^{\prime}\right)\right|^{2}\right)^{\frac{1}{2}}
$$

where $S^{(k, \alpha)}:=\prod_{j=1}^{k}\left\langle\nabla_{x_{j}}\right\rangle^{\alpha}\left\langle\nabla_{x_{j}^{\prime}}\right\rangle^{\alpha}$.

2.2. The GP hierarchy. We introduce cubic, quintic, focusing, and defocusing GP hierarchies, using the notation and definitions from [6].

Let $p \in\{2,4\}$. The $p$-GP (Gross-Pitaevskii) hierarchy is given by

$$
i \partial_{t} \gamma^{(k)}=\sum_{j=1}^{k}\left[-\Delta_{x_{j}}, \gamma^{(k)}\right]+\mu B_{k+\frac{p}{2}} \gamma^{\left(k+\frac{p}{2}\right)}
$$

in $d$ dimensions, for $k \in \mathbb{N}$. Here,

$$
B_{k+\frac{p}{2}} \gamma^{\left(k+\frac{p}{2}\right)}=B_{k+\frac{p}{2}}^{+} \gamma^{\left(k+\frac{p}{2}\right)}-B_{k+\frac{p}{2}}^{-} \gamma^{\left(k+\frac{p}{2}\right)},
$$

where

$$
B_{k+\frac{p}{2}}^{+} \gamma^{\left(k+\frac{p}{2}\right)}=\sum_{j=1}^{k} B_{j ; k+1, \ldots, k+\frac{p}{2}}^{+} \gamma^{\left(k+\frac{p}{2}\right)}
$$

and

$$
B_{k+\frac{p}{2}}^{-} \gamma^{\left(k+\frac{p}{2}\right)}=\sum_{j=1}^{k} B_{j ; k+1, \ldots, k+\frac{p}{2}}^{-} \gamma^{\left(k+\frac{p}{2}\right)}
$$


with

$$
\begin{aligned}
& \left(B_{j ; k+1, \ldots, k+\frac{p}{2}}^{+} \gamma^{\left(k+\frac{p}{2}\right)}\right)\left(t, x_{1}, \ldots, x_{k} ; x_{1}^{\prime}, \ldots, x_{k}^{\prime}\right) \\
& \quad=\int d x_{k+1} \cdots d x_{k+\frac{p}{2}} d x_{k+1}^{\prime} \cdots d x_{k+\frac{p}{2}}^{\prime} \\
& \quad \prod_{\ell=k+1}^{k+\frac{p}{2}} \delta\left(x_{j}-x_{\ell}\right) \delta\left(x_{j}-x_{\ell}^{\prime}\right) \gamma^{\left(k+\frac{p}{2}\right)}\left(t, x_{1}, \ldots, x_{k+\frac{p}{2}} ; x_{1}^{\prime}, \ldots, x_{k+\frac{p}{2}}^{\prime}\right),
\end{aligned}
$$

and

$$
\begin{aligned}
& \left(B_{j ; k+1, \ldots, k+\frac{p}{2}}^{-} \gamma^{\left(k+\frac{p}{2}\right)}\right)\left(t, x_{1}, \ldots, x_{k} ; x_{1}^{\prime}, \ldots, x_{k}^{\prime}\right) \\
& \quad=\int d x_{k+1} \cdots d x_{k+\frac{p}{2}} d x_{k+1}^{\prime} \cdots d x_{k+\frac{p}{2}}^{\prime} \\
& \quad \prod_{\ell=k+1}^{k+\frac{p}{2}} \delta\left(x_{j}^{\prime}-x_{\ell}\right) \delta\left(x_{j}^{\prime}-x_{\ell}^{\prime}\right) \gamma^{\left(k+\frac{p}{2}\right)}\left(t, x_{1}, \ldots, x_{k+\frac{p}{2}} ; x_{1}^{\prime}, \ldots, x_{k+\frac{p}{2}}^{\prime}\right) .
\end{aligned}
$$

The operator $B_{k+\frac{p}{2}} \gamma^{\left(k+\frac{p}{2}\right)}$ accounts for $\left(\frac{p}{2}+1\right)$-body interactions between the Bose particles. We remark that for factorized solutions

$$
\gamma^{(k)}\left(t, x_{1}, \ldots, x_{k} ; x_{1}^{\prime}, \ldots, x_{k}^{\prime}\right)=\prod_{j=1}^{k} \phi\left(t, x_{j}\right) \bar{\phi}\left(t, x_{j}^{\prime}\right),
$$

the corresponding 1-particle wave function satisfies the $p$-NLS

$$
i \partial_{t} \phi=-\Delta \phi+\mu|\phi|^{p} \phi
$$

which is focusing if $\mu=-1$, and defocusing if $\mu=+1$.

As in [6, 7], we refer to (2.5) as the cubic GP hierarchy if $p=2$, and as the quintic GP hierarchy if $p=4$. For $\mu=1$ or $\mu=-1$ we refer to the corresponding GP hierarchies as being defocusing or focusing, respectively.

The $p$-GP hierarchy can be rewritten in the following compact manner:

$$
\begin{aligned}
i \partial_{t} \Gamma+\widehat{\Delta}_{ \pm} \Gamma & =\mu \widehat{B} \Gamma, \\
\Gamma(0) & =\Gamma_{0},
\end{aligned}
$$

where

$$
\widehat{\Delta}_{ \pm} \Gamma:=\left(\Delta_{ \pm}^{(k)} \gamma^{(k)}\right)_{k \in \mathbb{N}}, \quad \text { with } \Delta_{ \pm}^{(k)}=\sum_{j=1}^{k}\left(\Delta_{x_{j}}-\Delta_{x_{j}^{\prime}}\right)
$$

and

$$
\widehat{B} \Gamma:=\left(B_{k+\frac{p}{2}} \gamma^{\left(k+\frac{p}{2}\right)}\right)_{k \in \mathbb{N}} .
$$

Also in this paper we will use the notation

$$
\begin{aligned}
& \widehat{B}^{+} \Gamma:=\left(B_{k+\frac{p}{2}}^{+} \gamma^{\left(k+\frac{p}{2}\right)}\right)_{k \in \mathbb{N}}, \\
& \widehat{B}^{-} \Gamma:=\left(B_{k+\frac{p}{2}}^{-} \gamma^{\left(k+\frac{p}{2}\right)}\right)_{k \in \mathbb{N}} .
\end{aligned}
$$

We refer to [6] for more detailed explanations. 


\section{Statement and proof of the main theorem}

In our earlier work [6], we proved local existence and uniqueness of solutions to the $p$-GP hierarchy in the space of solutions

$$
\mathcal{W}_{\xi}^{\alpha}(I):=\left\{\Gamma \mid \Gamma \in L_{t \in I}^{\infty} \mathcal{H}_{\xi}^{\alpha}, \widehat{B}^{+} \Gamma, \widehat{B}^{-} \Gamma \in L_{t \in I}^{2} \mathcal{H}_{\xi}^{\alpha}\right\}
$$

where $I:=[0, T]$. The requirement on the spacetime norm of $\widehat{B}^{ \pm} \Gamma$ corresponds to the Klainerman-Machedon a priori condition used in [19]. For our proof, we used a contraction-mapping argument, based on which both existence and uniqueness were obtained in the same process. However, the question remained whether the condition that $\widehat{B} \Gamma \in L_{t \in I}^{2} \mathcal{H}_{\xi}^{\alpha}$ for some $\xi$ is necessary for both the existence and uniqueness of solutions. In the present paper, we prove that for the existence part, this a priori assumption is not required. However, as an a posteriori result, we show that the solution obtained in this paper has the property that $\widehat{B} \Gamma \in L_{t \in I}^{2} \mathcal{H}_{\xi}^{\alpha}$.

We remark that for regularity $\alpha>\frac{d}{2}$, the result of this paper follows in an easier way by employing the estimate

$$
\|\widehat{B} \Gamma\|_{\mathcal{H}_{\xi}^{\alpha}} \leq C\|\Gamma\|_{\mathcal{H}_{\xi}^{\alpha}}
$$

instead of Strichartz estimates, which do not need to be invoked. The bound (3.2) for quintic GP was proved in our earlier work [5] (see Theorem 4.3), and was employed to give a short proof of uniqueness for the quintic GP hierarchy (see Theorem 6.1 in [5]). A bound of the type (3.2) for the cubic GP was proved in a recent paper of Chen and Liu [8] and was used to prove local well-posedness for the GP hierarchy in the case when $\alpha>\frac{d}{2}$.

Theorem 3.1. Let $\alpha \in \mathfrak{A}(d, p)$, where

$$
\mathfrak{A}(d, p):=\left\{\begin{array}{cc}
\left(\frac{1}{2}, \infty\right) & \text { if } d=1 \\
\left(\frac{d}{2}-\frac{1}{2(p-1)}, \infty\right) & \text { if } d \geq 2 \text { and }(d, p) \neq(3,2) \\
{[1, \infty)} & \text { if }(d, p)=(3,2),
\end{array}\right.
$$

and let $U(t)$ denote the free evolution $U(t)=e^{i t \widehat{\Delta}_{ \pm}}$. Assume that $\Gamma_{0} \in \mathcal{H}_{\xi^{\prime}}^{\alpha}$. Then, there exists a solution of the $p$-GP hierarchy $\Gamma \in L_{t \in I}^{\infty} \mathcal{H}_{\xi}^{\alpha}$ satisfying

$$
\Gamma(t)=U(t) \Gamma_{0}+i \mu \int_{0}^{t} U(t-s) \widehat{B} \Gamma(s) d s
$$

for $0<\xi<\xi^{\prime}$ sufficiently small (it is sufficient that $\xi<\eta^{2} \xi^{\prime}$, where the constant $\eta$ is specified in Lemma A.4 below).

In particular, this solution has the property that $\widehat{B} \Gamma \in L_{t \in I}^{2} \mathcal{H}_{\xi}^{\alpha}$.

Remark 3.2. We note that the presence of two different energy scales $\xi, \xi^{\prime}$ has the following interpretation on the level of the NLS. Let $R_{0}:=\left(\xi^{\prime}\right)^{-1 / 2}$ and $R_{1}:=$ $\xi^{-1 / 2}$. Then, the existence result in Theorem 3.1, applied to factorized initial data $\Gamma_{0}=\Gamma_{\phi_{0}}$ and the associated solution $\Gamma(t)=\Gamma_{\phi(t)}$ (of the form (2.7)), is equivalent to the following statement: For $\left\|\phi_{0}\right\|_{H^{1}\left(\mathbb{R}^{n}\right)}<R_{0}$, there exists a solution $\|\phi\|_{L_{t \in I}^{\infty} H^{1}\left(\mathbb{R}^{n}\right)}<R_{1}$, with $R_{1}>R_{0}$, in the space

$$
\left\{\phi \in L_{t \in I}^{\infty} H^{1}\left(\mathbb{R}^{n}\right)\left|\left\||\phi|^{p} \phi\right\|_{L_{t}^{2} H^{1}}<\infty\right\} .\right.
$$


This statement, specified for balls $B_{R_{0}}(0), B_{R_{1}}(0) \subset H^{1}\left(\mathbb{R}^{n}\right)$, contains the usual less specific formulation where only finiteness is required, that is, $\left\|\phi_{0}\right\|_{H^{1}\left(\mathbb{R}^{n}\right)}<\infty$ and $\|\phi\|_{L_{t \in I}^{\infty} H^{1}\left(\mathbb{R}^{n}\right)}<\infty$.

Proof. The $p$-GP hierarchy is given by

$$
i \partial_{t} \gamma^{(n)}=\sum_{j=1}^{n}\left[-\Delta_{x_{j}}, \gamma^{(n)}\right]+\mu B_{n+\frac{p}{2}} \gamma^{\left(n+\frac{p}{2}\right)}
$$

for all $n \in \mathbb{N}$.

We observe that (3.5) determines a closed, infinite subhierarchy, for initial data $\gamma_{N}^{(n)}(0)=0$, for $n>N$, which has the trivial solution

$$
\gamma_{N}^{(n)}(t)=0, \quad t \in I=[0, T], \quad n>N .
$$

Without invoking uniqueness, it is not possible to conclude that this is the unique solution of the subhierarchy for $n>N$ with zero initial data.

However, for the construction of a solution of (3.5) (without any statement on uniqueness), we are free to choose $\gamma_{N}^{(n)}(t)=0$ for $n>N$. In particular, it then follows that for $n \geq N-\frac{p}{2}+1$,

$$
i \partial_{t} \gamma_{N}^{(n)}\left(t ; \underline{x}_{N} ; \underline{x}_{N}^{\prime}\right)=\sum_{j=1}^{k}\left[-\Delta_{x_{j}}, \gamma_{N}^{(n)}\right]\left(t ; \underline{x}_{N} ; \underline{x}_{N}^{\prime}\right)
$$

solves the free evolution equation, since $B_{n+\frac{p}{2}} \gamma^{\left(n+\frac{p}{2}\right)}=0$. Thus,

$$
\gamma_{N}^{(n)}(t)=U^{(n)}(t) \gamma_{N}^{(n)}(0) \quad \text { for } n \geq N-\frac{p}{2}+1 .
$$

On the other hand, for $n \leq N-\frac{p}{2}, \gamma_{N}^{(n)}(t)$ satisfies the $p$-GP hierarchy in the full form (3.5).

From now on, for a fixed $N$, we consider solutions of (3.5) of the above type which we denote by $\Gamma_{N}=\left(\gamma_{N}^{(n)}\right)$. More precisely, let $P_{\leq N}$ denote the projection operator

$$
\begin{aligned}
P_{\leq N}: \mathfrak{G} & \rightarrow \mathfrak{G} \\
\Gamma=\left(\gamma^{(1)}, \gamma^{(2)}, \ldots\right) & \mapsto\left(\gamma^{(1)}, \ldots, \gamma^{(N)}, 0,0, \ldots\right),
\end{aligned}
$$

and $P_{>N}=1-P_{\leq N}$. We consider solutions $\Gamma_{N}(t)$ of the $p$-GP hierarchy,

$$
i \partial_{t} \Gamma_{N}=\widehat{\Delta}_{ \pm} \Gamma_{N}+\mu \widehat{B} \Gamma_{N}
$$

for the truncated initial data

$$
\Gamma_{N}(0)=P_{\leq N} \Gamma_{0}=\left(\gamma_{0}^{(1)}, \ldots, \gamma_{0}^{(N)}, 0,0, \ldots\right)
$$

for an arbitrary, large, fixed $N \in \mathbb{N}$, and where the component $\Gamma_{N}^{(m)}(t)=0$ for the $m$-th component, for all $m>N$. By Duhamel's formula, the solution of (3.10) is given by

$$
\Gamma_{N}(t)=U(t) \Gamma_{N}(0)+i \mu \int_{0}^{t} U(t-s) \widehat{B} \Gamma_{N}(s) d s
$$

for initial data $\Gamma_{N}(0)=P_{\leq N} \Gamma_{0}$. 
We introduce three parameters $\xi, \xi^{\prime \prime}, \xi^{\prime}$ satisfying

$$
\xi<\eta \xi^{\prime \prime}<\eta^{2} \xi^{\prime}
$$

where the constant $0<\eta<1$ is specified in Lemma A.4 below.

It is shown in Appendix $\mathrm{B}$ that it is sufficient to iterate the Duhamel formula (3.12) for $\Gamma_{N}$ only finitely many times, in order to obtain a fully explicit solution to (3.10) for fixed $N$ that satisfies

$$
\left\|\Gamma_{N}\right\|_{L_{t \in I}^{\infty} \mathcal{H}_{\xi^{\prime \prime}}^{\alpha}}^{\alpha}, \quad\left\|\widehat{B} \Gamma_{N}\right\|_{L_{t \in I}^{2} \mathcal{H}_{\xi^{\prime \prime}}^{\alpha}} \leq C\left(T, \xi^{\prime}, \xi^{\prime \prime}\right)\left\|\Gamma_{0}\right\|_{\mathcal{H}_{\xi^{\prime}}^{\alpha}} .
$$

Moreover, it follows from Lemma A.4 below that the sequence $\left(\widehat{B} \Gamma_{N}\right)_{N \in \mathbb{N}}$ is Cauchy in $L_{t \in I}^{2} \mathcal{H}_{\xi^{\prime \prime}}^{\alpha}$. That is, for any $\epsilon>0$, there exists $N(\epsilon) \in \mathbb{N}$ such that

$$
\begin{aligned}
\left\|\widehat{B}\left(\Gamma_{N_{1}}-\Gamma_{N_{2}}\right)\right\|_{L_{t \in I}^{2} \mathcal{H}_{\xi^{\prime \prime}}^{\alpha}} & \leq C\left(T, \xi^{\prime}, \xi^{\prime \prime}\right)\left\|P_{>N_{1}} \Gamma_{0}\right\|_{\mathcal{H}_{\xi^{\prime}}^{\alpha}} \\
& <\epsilon
\end{aligned}
$$

holds for all $N_{1}, N_{2}>N(\epsilon)$. This is because by assumption, $\left\|\Gamma_{0}\right\|_{\mathcal{H}_{\xi^{\prime}}^{\alpha}}<\infty$, which is a power series in $\xi^{\prime}>0$ with nonnegative coefficients. Hence,

$$
\left\|P_{>N_{1}} \Gamma_{0}\right\|_{\mathcal{H}_{\xi^{\prime}}^{\alpha}}=\sum_{k>N_{1}}\left(\xi^{\prime}\right)^{k}\left\|\gamma^{(k)}(0)\right\|_{H^{\alpha}} \rightarrow 0
$$

as $N_{1} \rightarrow \infty$, so that (3.16) follows.

Accordingly, there exists a strong limit

$$
\Theta=\lim _{N \rightarrow \infty} \widehat{B} \Gamma_{N} \in L_{t \in I}^{2} \mathcal{H}_{\xi^{\prime \prime}}^{\alpha}
$$

We claim that

$$
\Theta(t)=\widehat{B} U(t) \Gamma(0)+i \mu \int_{0}^{t} \widehat{B} U(t-s) \Theta(s) d s .
$$

In order to prove this claim, we observe that

$$
\begin{aligned}
\| \Theta(t) & -\widehat{B} U(t) \Gamma(0)-i \mu \int_{0}^{t} \widehat{B} U(t-s) \Theta(s) \|_{L_{t \in I}^{2} \mathcal{H}_{\xi}^{\alpha}} \\
\leq \quad & \Theta(t)-\widehat{B} \Gamma_{N}(t) \|_{L_{t \in I}^{2} \mathcal{H}_{\xi}^{\alpha}} \\
+ & \left\|\widehat{B} U(t)\left(\Gamma(0)-\Gamma_{N}(0)\right)\right\|_{L_{t \in I}^{2} \mathcal{H}_{\xi}^{\alpha}} \\
+ & \left\|\int_{0}^{t} d s \widehat{B} U(t-s)\left(\Theta(s)-\widehat{B} \Gamma_{N}(s)\right)\right\|_{L_{t \in I}^{2} \mathcal{H}_{\xi}^{\alpha}} \\
+ & \left\|\widehat{B} \Gamma_{N}(t)-\widehat{B} U(t) \Gamma_{N}(0)-i \int_{0}^{t} \widehat{B} U(t-s) \widehat{B} \Gamma_{N}(s) d s\right\|_{L_{t \in I}^{2} \mathcal{H}_{\xi}^{\alpha}} .
\end{aligned}
$$

First, we notice that (3.23) is identically zero because $\Gamma_{N}$ is a solution of the $p$-GP hierarchy, (3.5). Since $\xi<\xi^{\prime \prime}$ we can estimate the term (3.20) as follows:

$$
\begin{gathered}
\left\|\Theta(t)-\widehat{B} \Gamma_{N}(t)\right\|_{L_{t \in I}^{2} \mathcal{H}_{\xi}^{\alpha}} \leq\left\|\Theta(t)-\widehat{B} \Gamma_{N}(t)\right\|_{L_{t \in I}^{2} \mathcal{H}_{\xi^{\prime \prime}}^{\alpha}} \\
=o_{N}(1),
\end{gathered}
$$


where the last line follows from (3.18). For (3.21), since $\xi<\eta \xi^{\prime \prime}$ we can use the free Strichartz estimate (A.1) as follows:

$$
\begin{aligned}
\left\|\widehat{B} U(t)\left(\Gamma(0)-\Gamma_{N}(0)\right)\right\|_{L_{t \in I}^{2} \mathcal{H}_{\xi}^{\alpha}} & \leq\left\|\Gamma(0)-\Gamma_{N}(0)\right\|_{\mathcal{H}_{\xi^{\prime \prime}}^{\alpha}} \\
& \leq\left\|\Gamma(0)-\Gamma_{N}(0)\right\|_{\mathcal{H}_{\xi^{\prime}}^{\alpha}} \\
& =\left\|P_{>N} \Gamma(0)\right\|_{\mathcal{H}_{\xi^{\prime}}^{\alpha}} .
\end{aligned}
$$

For the term (3.22), we have

$$
\begin{aligned}
& \left\|\int_{0}^{t} d s \widehat{B} U(t-s)\left(\Theta(s)-\widehat{B} \Gamma_{N}(s)\right)\right\|_{L_{t \in I}^{2} \mathcal{H}_{\xi}^{\alpha}} \\
& \quad \leq\left\|\int_{0}^{t} d s\right\| \widehat{B} U(t-s)\left(\Theta(s)-\widehat{B} \Gamma_{N}(s)\right)\left\|_{\mathcal{H}_{\xi}^{\alpha}}\right\|_{L_{t \in I}^{2}} \\
& \quad \leq \int_{0}^{T} d s\left\|\widehat{B} U(t-s)\left(\Theta(s)-\widehat{B} \Gamma_{N}(s)\right)\right\|_{L_{t \in I}^{2} \mathcal{H}_{\xi}^{\alpha}} \\
& \quad \leq C\left(T, \xi, \xi^{\prime \prime}\right) \int_{0}^{T} d s\left\|\Theta(s)-\widehat{B} \Gamma_{N}(s)\right\|_{\mathcal{H}_{\xi^{\prime \prime}}^{\alpha}} \\
& \quad \leq C\left(T, \xi, \xi^{\prime \prime}\right) T^{1 / 2}\left\|\Theta(s)-\widehat{B} \Gamma_{N}(s)\right\|_{L_{t \in I}^{2} \mathcal{H}_{\xi^{\prime \prime}}^{\alpha}} \\
& \quad=o_{N}(1) .
\end{aligned}
$$

Here, to obtain (3.26) we use the free Strichartz estimate A.1 in a manner similar to the $T-T^{*}$ argument for the Schrödinger equation. To obtain (3.27) we used the Hölder inequality, and to get the last line (3.28), we used (3.18). In conclusion,

$$
\begin{gathered}
\left\|\Theta(t)-\widehat{B} U(t) \Gamma(0)-i \mu \int_{0}^{t} d s \widehat{B} U(t-s) \Theta(s)\right\|_{L_{t \in I}^{2} \mathcal{H}_{\xi}^{\alpha}} \\
\leq o_{N}(1)+C(T, \xi)\left\|P_{>N} \Gamma(0)\right\|_{\mathcal{H}_{\xi^{\prime}}^{\alpha}} \rightarrow 0 \quad(N \rightarrow \infty) .
\end{gathered}
$$

Therefore, taking the limit $N \rightarrow \infty$, we find that $\Theta$ satisfies

$$
\Theta(t)=\widehat{B} U(t) \Gamma(0)+i \mu \int_{0}^{t} \widehat{B} U(t-s) \Theta(s) d s
$$

as claimed.

Moreover, we observe that for $t \in I=[0, T]$ we have:

$$
\begin{aligned}
& \left\|\Gamma_{N_{1}}(t)-\Gamma_{N_{2}}(t)\right\|_{\mathcal{H}_{\xi}^{\alpha}} \\
& \leq\left\|U(t)\left(\Gamma_{N_{1}}(0)-\Gamma_{N_{2}}(0)\right)\right\|_{\mathcal{H}_{\xi}^{\alpha}}+\left\|\int_{0}^{t} d s U(t-s) \widehat{B}\left(\Gamma_{N_{1}}(s)-\Gamma_{N_{2}}(s)\right)\right\|_{\mathcal{H}_{\xi}^{\alpha}} \\
& \leq\left\|\Gamma_{N_{1}}(0)-\Gamma_{N_{2}}(0)\right\|_{\mathcal{H}_{\xi}^{\alpha}}+\int_{0}^{T} d s\left\|\widehat{B}\left(\Gamma_{N_{1}}(s)-\Gamma_{N_{2}}(s)\right)\right\|_{\mathcal{H}_{\xi}^{\alpha}} \\
& \leq\left\|\Gamma_{N_{1}}(0)-\Gamma_{N_{2}}(0)\right\|_{\mathcal{H}_{\xi^{\prime}}^{\alpha}}+\int_{0}^{T} d s\left\|\widehat{B}\left(\Gamma_{N_{1}}(s)-\Gamma_{N_{2}}(s)\right)\right\|_{\mathcal{H}_{\xi^{\prime \prime}}^{\alpha}} \\
& \leq\left\|\Gamma_{N_{1}}(0)-\Gamma_{N_{2}}(0)\right\|_{\mathcal{H}_{\xi^{\prime}}^{\alpha}}+T^{\frac{1}{2}}\left\|\widehat{B}\left(\Gamma_{N_{1}}(s)-\Gamma_{N_{2}}(s)\right)\right\|_{L_{s \in I}^{2} \mathcal{H}_{\xi^{\prime \prime}}} \\
& \leq C\left(T, \xi^{\prime}, \xi^{\prime \prime}\right)\left\|P_{>N_{1}} \Gamma_{0}\right\|_{\mathcal{H}_{\xi^{\prime}}^{\alpha}},
\end{aligned}
$$


using the relation $\xi<\eta \xi^{\prime \prime}<\eta^{2} \xi^{\prime}$ to obtain (3.31), and (3.15) to pass to the last line. Thus, similarly as in (3.16), there exists for every $\epsilon>0$ a number $N(\epsilon) \in \mathbb{N}$ such that

$$
\left\|\Gamma_{N_{1}}(t)-\Gamma_{N_{2}}(t)\right\|_{\mathcal{H}_{\xi}^{\alpha}}<\epsilon
$$

for all $N_{1}, N_{2}>N(\epsilon)$. This implies that $\left(\Gamma_{N}\right)_{N \in \mathbb{N}}$ is a Cauchy sequence in $L_{t \in I}^{\infty} \mathcal{H}_{\xi}^{\alpha}$; thus we obtain the strong limit

$$
\Gamma=\lim _{N \rightarrow \infty} \Gamma_{N} \in L_{t \in I}^{\infty} \mathcal{H}_{\xi}^{\alpha},
$$

given the initial data $\Gamma_{0} \in \mathcal{H}_{\xi^{\prime}}^{\alpha}$.

Next, we claim that $\Gamma$ satisfies

$$
\Gamma(t)=U(t) \Gamma_{0}+i \mu \int_{0}^{t} U(t-s) \Theta(s) d s .
$$

Indeed, we have for $t \in I$ that

$$
\begin{aligned}
\| \Gamma(t) & -U(t) \Gamma_{0}-i \mu \int_{0}^{t} U(t-s) \Theta(s) d s \|_{\mathcal{H}_{\xi}^{\alpha}} \\
\leq \quad & \left\|\Gamma(t)-\Gamma_{N}(t)\right\|_{\mathcal{H}_{\xi}^{\alpha}} \\
& +\left\|U(t)\left(\Gamma_{0}-\Gamma_{N}(0)\right)\right\|_{\mathcal{H}_{\xi}^{\alpha}} \\
& +\left\|\int_{0}^{t} U(t-s)\left(\Theta(s)-\widehat{B} \Gamma_{N}(s)\right) d s\right\|_{\mathcal{H}_{\xi}^{\alpha}} \\
& +\left\|\Gamma_{N}(t)-U(t) \Gamma_{N}(0)-i \mu \int_{0}^{t} U(t-s) \widehat{B} \Gamma_{N}(s) d s\right\|_{\mathcal{H}_{\xi}^{\alpha}} .
\end{aligned}
$$

Here, we note that (3.38) is identically zero because $\Gamma_{N}(t)$ is a solution of the $p$-GP hierarchy, (3.5). Moreover, we have

$$
\begin{aligned}
\text { (3.37) } & \leq \int_{0}^{T} d s\left\|\Theta(s)-\widehat{B} \Gamma_{N}(s)\right\|_{\mathcal{H}_{\xi}^{\alpha}} \\
& \leq T^{\frac{1}{2}}\left\|\Theta-\widehat{B} \Gamma_{N}\right\|_{L_{t \in I}^{2} \mathcal{H}_{\xi}^{\alpha}} \\
& \leq T^{\frac{1}{2}}\left\|\Theta-\widehat{B} \Gamma_{N}\right\|_{L_{t \in I}^{2} \mathcal{H}_{\xi^{\prime \prime}}^{\alpha}} .
\end{aligned}
$$

Therefore,

$$
\begin{gathered}
\left\|\Gamma(t)-U(t) \Gamma_{0}-i \mu \int_{0}^{t} U(t-s) \Theta(s) d s\right\|_{L_{t \in I}^{\infty} \mathcal{H}_{\xi}^{\alpha}} \\
(3.40) \leq\left\|\Gamma-\Gamma_{N}\right\|_{L_{t \in I}^{\infty} \mathcal{H}_{\xi}^{\alpha}}+\left\|\Gamma_{0}-\Gamma_{N}(0)\right\|_{\mathcal{H}_{\xi}^{\alpha}}+T^{\frac{1}{2}}\left\|\Theta-\widehat{B} \Gamma_{N}\right\|_{L_{t \in I}^{2} \mathcal{H}_{\xi^{\prime \prime}}^{\alpha}},
\end{gathered}
$$

where the right-hand side tends to zero as $N \rightarrow \infty$, due to the convergence (3.33) and (3.18). This implies (3.34).

Finally, we observe that

$$
\widehat{B} \Gamma(t)=\widehat{B} U(t) \Gamma_{0}+i \mu \int_{0}^{t} \widehat{B} U(t-s) \Theta(s) d s
$$

while

$$
\Theta(t)=\widehat{B} U(t) \Gamma_{0}+i \mu \int_{0}^{t} \widehat{B} U(t-s) \Theta(s) d s .
$$


Comparing the right-hand sides, we infer that $\widehat{B} \Gamma=\Theta \in L_{t \in I}^{2} \mathcal{H}_{\xi}^{\alpha}$. This concludes the proof.

\section{Appendix A. Iterated Duhamel formula and boardgame argument}

In this section, our main goal is to prove Lemma A.4 below; it is the main ingredient for establishing that $\left(\widehat{B} \Gamma_{N}\right)$ is a Cauchy sequence in $L_{t \in I}^{2} \mathcal{H}_{\xi}^{\alpha}$. We first summarize some results established in [5, 6, 19, which are related to Strichartz estimates for the GP hierarchy.

We first reformulate the Strichartz estimate for the free evolution $U(t)=e^{i t \widehat{\Delta}_{ \pm}}=$ $\left(U^{(n)}(t)\right)_{n \in \mathbb{N}}$ proven in [6, 19].

Lemma A.1. Let $\alpha \in \mathfrak{A}(d, p)$. Assume that $\Gamma_{0} \in \mathcal{H}_{\xi^{\prime}}^{\alpha}$ for some $0<\xi^{\prime}<1$. Then, for any $0<\xi<\xi^{\prime}$, there exists a constant $C\left(\xi, \xi^{\prime}\right)$ such that the Strichartz estimate for the free evolution

$$
\left\|\widehat{B} U(t) \Gamma_{0}\right\|_{L_{t \in \mathbb{R}}^{2} \mathcal{H}_{\xi}^{\alpha}} \leq C\left(\xi, \xi^{\prime}\right)\left\|\Gamma_{0}\right\|_{\mathcal{H}_{\xi^{\prime}}^{\alpha}}
$$

holds.

Proof. From Theorem 1.3 in [19] and Proposition A.1 in [6], we have, for $\alpha \in \mathfrak{A}(d, p)$, that

$$
\begin{aligned}
& \left\|B^{\left(k+\frac{p}{2}\right)} U^{\left(k+\frac{p}{2}\right)}(t) \gamma_{0}^{\left(k+\frac{p}{2}\right)}\right\|_{L_{t \in \mathbb{R}}^{2} H_{k}^{\alpha}} \\
& \quad \leq 2 \sum_{j=1}^{k}\left\|B_{j ; k+1, \ldots, k+\frac{p}{2}}^{+} U^{\left(k+\frac{p}{2}\right)}(t) \gamma_{0}^{\left(k+\frac{p}{2}\right)}\right\|_{L_{t \in \mathbb{R}}^{2} H_{k}^{\alpha}} \\
& \quad \leq C k\left\|\gamma_{0}^{\left(k+\frac{p}{2}\right)}\right\|_{H_{k+\frac{p}{2}}^{\alpha}} .
\end{aligned}
$$

Then for any $0<\xi<\xi^{\prime}$, we have

$$
\begin{aligned}
\left\|\widehat{B} U(t) \Gamma_{0}\right\|_{L_{t \in \mathbb{R}}^{2} \mathcal{H}_{\xi}^{\alpha}} & \leq \sum_{k \geq 1} \xi^{k}\left\|B^{\left(k+\frac{p}{2}\right)} U^{\left(k+\frac{p}{2}\right)}(t) \gamma_{0}^{\left(k+\frac{p}{2}\right)}\right\|_{L_{t \in \mathbb{R}}^{2} H_{k}^{\alpha}} \\
& \leq C \sum_{k \geq 1} k \xi^{k}\left\|\gamma_{0}^{\left(k+\frac{p}{2}\right)}\right\|_{H_{k+\frac{p}{2}}^{\alpha}} \\
& =C\left(\xi^{\prime}\right)^{-\frac{p}{2}} \sum_{k \geq 1} k\left(\frac{\xi}{\xi^{\prime}}\right)^{k}\left(\xi^{\prime}\right)^{\left(k+\frac{p}{2}\right)}\left\|\gamma_{0}^{\left(k+\frac{p}{2}\right)}\right\|_{H_{k+\frac{p}{2}}^{\alpha}} \\
& \leq C\left(\xi^{\prime}\right)^{-\frac{p}{2}} \sup _{k \geq 1} k\left(\frac{\xi}{\xi^{\prime}}\right)^{k} \sum_{k \geq 1}\left(\xi^{\prime}\right)^{\left(k+\frac{p}{2}\right)}\left\|\gamma_{0}^{\left(k+\frac{p}{2}\right)}\right\|_{H_{k+\frac{p}{2}}^{\alpha}} \\
& \leq C\left(\xi, \xi^{\prime}\right)\left\|\Gamma_{0}\right\|_{\mathcal{H}_{\xi^{\prime}}^{\alpha}},
\end{aligned}
$$

where to obtain (A.3) we used (A.2).

Definition A.2. Let $\widetilde{\Gamma}=\left(\widetilde{\gamma}^{(n)}\right)_{n \in \mathbb{N}}$ denote a sequence of arbitrary Schwartz class functions $\widetilde{\gamma}^{(n)} \in \mathcal{S}\left(\mathbb{R} \times \mathbb{R}^{n d} \times \mathbb{R}^{n d}\right)$. Then, we define the associated sequence $\operatorname{Duh}_{j}(\widetilde{\Gamma})$ 
of $j$-th level iterated Duhamel terms, with $n$-th component given by

$$
\begin{array}{r}
\operatorname{Duh}_{j}(\widetilde{\Gamma})^{(n)}(t):=(-i \mu)^{j} \int_{0}^{t} d t_{1} \cdots \int_{0}^{t_{j-1}} d t_{j} e^{i\left(t-t_{1}\right) \Delta_{ \pm}^{(n)}} B_{n+\frac{p}{2}} e^{i\left(t_{1}-t_{2}\right) \Delta_{ \pm}^{\left(n+\frac{p}{2}\right)}} \\
B_{n+\frac{2 p}{2}} \cdots \cdots B_{n+\frac{j p}{2}} e^{i t_{j} \Delta_{ \pm}^{\left(n+\frac{j p}{2}\right)}} \widetilde{\gamma}^{\left(n+\frac{j p}{2}\right)}\left(t_{j}\right)
\end{array}
$$

and $\operatorname{Duh}_{0}(\widetilde{\Gamma})^{(n)}(t):=U^{(n)}(t) \widetilde{\gamma}^{(n)}(t)$, for $j \in \mathbb{N}_{0}$.

As usual, the definition is given for Schwartz class functions and can be extended to the spaces in discussion by density arguments. The fact that $\operatorname{Duh}_{j}(\widetilde{\Gamma})^{(n)} \in$ $\mathcal{S}\left(\mathbb{R} \times \mathbb{R}^{n d} \times \mathbb{R}^{n d}\right)$ holds under the above conditions, for all $n$, can be easily verified. Using the boardgame strategy of [19] (which is a reformulation of a combinatorial argument developed in [10, 11]), one obtains:

Lemma A.3. Let $\alpha \in \mathfrak{A}(d, p)$ and $p \in\{2,4\}$. Then, for $\widetilde{\Gamma}=\left(\widetilde{\gamma}^{(n)}\right)_{n \in \mathbb{N}}$ as above,

$$
\begin{aligned}
& \left\|B_{n+\frac{p}{2}} \operatorname{Duh}_{j}(\widetilde{\Gamma})^{\left(n+\frac{p}{2}\right)}(t)\right\|_{L_{t \in I}^{2} H^{\alpha}\left(\mathbb{R}^{n d} \times \mathbb{R}^{n d}\right)} \\
& \quad \leq n C_{0}^{n}\left(c_{0} T\right)^{\frac{j}{2}}\left\|B_{n+\frac{(j+1) p}{2}} U^{\left(n+\frac{(j+1) p}{2}\right)}(\cdot) \widetilde{\gamma}^{\left(n+\frac{(j+1) p}{2}\right)}\right\|_{L_{t \in I}^{2} H^{\alpha}\left(\mathbb{R}^{\left(n+\frac{j p}{2}\right) d} \times \mathbb{R}^{\left(n+\frac{j p}{2}\right) d}\right)},
\end{aligned}
$$

where the constants $c_{0}, C_{0}$ depend only on $d, p$.

For the proof in the cubic case, $p=2$, we refer to [6, 19, and for the proof in the quintic case, $p=4$, to $[5]$.

We observe that any solution $\Gamma_{N}$ of (3.5) with initial data $\Gamma_{N}(0)=P_{\leq N} \Gamma_{0}$ satisfies the equation

$$
\widehat{B} \Gamma_{N}(t)=\widehat{B} U(t) \Gamma_{N}(0)+i \int_{0}^{t} \widehat{B} U(t-s) \widehat{B} \Gamma_{N}(s) d s
$$

(obtained from acting with $\widehat{B}$ on $(3.12)$ ), and by recursion,

$$
\begin{aligned}
\left(\widehat{B} \Gamma_{N}\right)^{(n)}(t)=\sum_{j=0}^{k-1} B_{n+\frac{p}{2}} \operatorname{Duh}_{j}\left(\Gamma_{N}(0)\right)^{\left(n+\frac{p}{2}\right)}(t) & \\
& +B_{n+\frac{p}{2}} \operatorname{Duh}_{k}\left(\widehat{B} \Gamma_{N}\right)^{\left(n+\frac{p}{2}\right)}(t),
\end{aligned}
$$

obtained from iterating the Duhamel formula $k$ times for the $n$-th component of $\widehat{B} \Gamma$ (for the definition of $\mathrm{Duh}_{j}$, see (A.4) $)$. Since $\Gamma_{N}^{(m)}(t)=0$ for all $m>N$, the remainder term on the last line is zero whenever $n+\frac{(k+1) p}{2}>N$. Thus,

$$
\left(\widehat{B} \Gamma_{N}\right)^{(n)}(t)=\sum_{j=0}^{\lceil 2(N-n) / p-1\rceil} B_{n+\frac{p}{2}} \operatorname{Duh}_{j}\left(\Gamma_{N}(0)\right)^{\left(n+\frac{p}{2}\right)}(t),
$$

where each term on the right explicitly depends only on the initial data $\Gamma_{N}(0)$ (there is no implicit dependence on the solution $\Gamma_{N}(t)$ ). 
Now we are ready to prove the main result of this section:

Lemma A.4. Assume that $\alpha \in \mathfrak{A}(d, p)$ and $p \in\{2,4\}$, and that $\Gamma_{0} \in \mathcal{H}_{\xi^{\prime}}^{\alpha}$ for some $0<\xi^{\prime}<1$. Let $N_{1}, N_{2} \in \mathbb{N}$, where $N_{1}<N_{2}$. Then, there exists a constant $0<\eta=\eta(d, p)<1$ such that the estimate

$$
\left\|\widehat{B}\left(\Gamma_{N_{1}}-\Gamma_{N_{2}}\right)\right\|_{L_{t \in I}^{2} \mathcal{H}_{\xi}^{\alpha}} \leq C\left(T, \xi, \xi^{\prime}\right)\left\|P_{>N_{1}} \Gamma_{0}\right\|_{\mathcal{H}_{\xi^{\prime}}^{\alpha}}
$$

holds whenever $\xi<\eta \xi^{\prime}$ (we note that it suffices to let $\eta<C_{0}^{-1}$, where the constant $C_{0}=C_{0}(d, p)$ is specified in Lemma A.3).

Proof. For simplicity of notation, we shall present the explicit arguments for the (cubic) case $p=2$. The (quintic) case $p=4$ is completely analogous.

Thanks to (A.8) we have

$$
\begin{aligned}
\left(\widehat{B}\left(\Gamma_{N_{1}}-\Gamma_{N_{2}}\right)\right)^{(n)}(t)= & \sum_{j=0}^{N_{1}-n-1} B_{n+1} \operatorname{Duh}_{j}\left(\left(\Gamma_{N_{1}}(0)-\Gamma_{N_{2}}(0)\right)^{(n+1)}(t)\right. \\
& -\sum_{j=N_{1}-n}^{N_{2}-n-1} B_{n+1} \operatorname{Duh}_{j}\left(\Gamma_{N_{2}}(0)\right)^{(n+1)}(t),
\end{aligned}
$$

using (A.4) and the fact that $\gamma_{N_{i}}^{(n+j+1)}=0$ for $j>N_{i}-n-1$, with $i=1,2$.

Since $\gamma_{N_{1}}^{(n+j+1)}(0)=\gamma_{N_{2}}^{(n+j+1)}(0)$ for $0 \leq j \leq N_{1}-n-1$, the first sum on the rhs of (A.10) is identically zero.

For the second term on the rhs of (A.10), we have for the summation index that $j \geq N_{1}-n$. Thus, the components of $\Gamma_{N_{2}}(0)$ occurring in (A.10) are given by $\gamma_{N_{2}}^{(n+j+1)}$ with $j \geq N_{1}-n$, that is, $\gamma_{N_{2}}^{(m)}$ with $m>N_{1}$.

Using Lemma A.3 and the free Strichartz estimate (A.2), we therefore find that

$$
\begin{aligned}
& \left\|\left(\widehat{B}\left(\Gamma_{N_{1}}-\Gamma_{N_{2}}\right)\right)^{(n)}(t)\right\|_{L_{t \in I}^{2} H^{\alpha}} \\
& \leq \sum_{j=N_{1}-n}^{N_{2}-n-1}\left\|B_{n+1} \operatorname{Duh}_{j}\left(\Gamma_{N_{2}}(0)\right)^{(n+1)}(t)\right\|_{L_{t \in I}^{2} H^{\alpha}} \\
& \leq \sum_{j=N_{1}-n}^{N_{2}-n-1} n C_{0}^{n}\left(c_{0} T\right)^{\frac{j}{2}}\left\|B_{n+j+1} U^{(n+j+1)}(t) \gamma_{N_{2}}^{(n+j+1)}(0)\right\|_{L_{t \in I}^{2} H^{\alpha}} \\
& \leq\left(c_{0} T\right)^{-\frac{1}{2}}\left(\xi^{\prime}\right)^{-n} n^{2} C_{0}^{n} \sum_{j=N_{1}-n}^{N_{2}-n-1}\left(c_{0} T\left(\xi^{\prime}\right)^{-2}\right)^{\frac{j+1}{2}}\left(\xi^{\prime}\right)^{n+j+1}\left\|\gamma_{N_{2}}^{(n+j+1)}(0)\right\|_{H^{\alpha}} \\
& \leq\left(\xi^{\prime}\right)^{-n} n^{2} C_{0}^{n} C_{1}\left(T, \xi^{\prime}\right)\left\|P_{>N_{1}} \Gamma_{N_{2}}(0)\right\|_{\mathcal{H}_{\xi^{\prime}}^{\alpha}},
\end{aligned}
$$

for $T>0$ sufficiently small so that $c_{0} T\left(\xi^{\prime}\right)^{-2} \leq 1$. Hence,

$$
\begin{aligned}
& \sum_{n \in \mathbb{N}} \xi^{n}\left\|\left(\widehat{B}\left(\Gamma_{N_{1}}-\Gamma_{N_{2}}\right)\right)^{(n)}(t)\right\|_{L_{t \in I}^{2} H^{\alpha}} \\
& \quad \leq C_{1}\left(T, \xi^{\prime}\right)\left(\sum_{n \in \mathbb{N}} n^{2} C_{0}^{n}\left(\xi / \xi^{\prime}\right)^{n}\right)\left\|P_{>N_{1}} \Gamma_{N_{2}}(0)\right\|_{\mathcal{H}_{\xi^{\prime}}^{\alpha}} \\
& \quad \leq C\left(T, \xi, \xi^{\prime}\right)\left\|P_{>N_{1}} \Gamma_{N_{2}}(0)\right\|_{\mathcal{H}_{\xi^{\prime}}^{\alpha}},
\end{aligned}
$$

for $\xi<\eta \xi^{\prime}$, where $\eta<C_{0}^{-1}$, noting that $C_{0}=C_{0}(d, p)$. 
This proves the claim for the case $p=2$. The case $p=4$ is completely analogous, and we shall omit a repetition of the arguments.

\section{Appendix B. Existence of SOlutions of the TRUnCATED HIERARCHY}

Having the additional notation and discussion presented in Appendix A at our disposal, we now return to discussing the existence of solutions to the $p$-GP hierarchy (3.10) with truncated initial data, for fixed $N$.

We recall that according to (A.8) we have:

$$
\left(\widehat{B} \Gamma_{N}\right)^{(n)}(t)=\sum_{j=0}^{\lceil 2(N-n) / p-1\rceil} B_{n+\frac{p}{2}} \operatorname{Duh}_{j}\left(\Gamma_{N}(0)\right)^{\left(n+\frac{p}{2}\right)}(t),
$$

where each term on the right explicitly depends only on the initial data $\Gamma_{N}(0)$.

Likewise, we find that the $n$-th component of $\Gamma_{N}(t)=\left(\gamma_{N}^{(n)}(t)\right)_{n}$ is given by the finite sum

$$
\gamma_{N}^{(n)}(t)=\sum_{j=0}^{\lceil 2(N-n) / p\rceil} \operatorname{Duh}_{j}\left(\Gamma_{0}\right)^{(n)}(t) .
$$

This is a fully explicit expression for a solution $\Gamma_{N}(t)$ of (3.5), obtained from only finitely many iterations of the Duhamel formula.

We next recall from (3.13) the triple of parameters $\xi, \xi^{\prime \prime}, \xi^{\prime}$ satisfying $\xi<\eta \xi^{\prime \prime}<$ $\eta^{2} \xi^{\prime}$, where the constant $0<\eta<1$ is specified in Lemma A.4 Combining Lemma A.3 with Lemma A.1 and repeating arguments similar to those presented in (A.11), we obtain that for $\alpha \in \mathfrak{A}(d, p)$ and $p \in\{2,4\}$, we have

$$
\left\|\widehat{B} \Gamma_{N}\right\|_{L_{t \in I}^{2} \mathcal{H}_{\xi^{\prime \prime}}^{\alpha}} \leq C\left(T, \xi^{\prime}, \xi^{\prime \prime}\right)\left\|\Gamma_{0}\right\|_{\mathcal{H}_{\xi^{\prime}}^{\alpha}} .
$$

We note that this result corresponds to A.11 for $N_{2}=N$ and $N_{1}=0$, up to the zeroth Duhamel term. Likewise, we obtain

$$
\left\|\Gamma_{N}\right\|_{L_{t \in I}^{\infty} \mathcal{H}_{\xi^{\prime \prime}}^{\alpha}} \leq C\left(T, \xi^{\prime}, \xi^{\prime \prime}\right)\left\|\Gamma_{0}\right\|_{\mathcal{H}_{\xi^{\prime}}^{\alpha}} .
$$

A detailed presentation of the proof of estimates of the type of (B.3) and (B.4) is given in [6], for the case where the Duhamel series does not terminate (due to initial data without cutoff).

We summarize that here, as opposed to the case treated in [6], the series (B.2) and (A.8) are finite, which is due to the truncation controlled by $N$. As a consequence, the proof of (B.3) does not require any additional a priori assumption on $\widehat{B} \Gamma_{N}$; the condition $\Gamma_{0} \in \mathcal{H}_{\xi^{\prime}}^{\alpha}$ is sufficient.

\section{ACKNOWLEDGEMENTS}

We are grateful to Igor Rodnianski for pointing out an error in an earlier version of this work and for useful comments. The work of the first author is supported by NSF grants DMS-0940145 and DMS-1009448. The work of the second author is supported by NSF grants number DMS 0758247 and DMS 1101192, and an Alfred P. Sloan Research Fellowship. 


\section{REFERENCES}

[1] R. Adami, G. Golse, A. Teta, Rigorous derivation of the cubic NLS in dimension one, J. Stat. Phys. 127, no. 6, 1194-1220 (2007). MR2331036 (2008i:82055)

[2] M. Aizenman, E.H. Lieb, R. Seiringer, J.P. Solovej, J. Yngvason, Bose-Einstein Quantum Phase Transition in an Optical Lattice Model, Phys. Rev. A 70, 023612 (2004).

[3] I. Anapolitanos, I.M. Sigal, The Hartree-von Neumann limit of many body dynamics, Preprint http://arxiv.org/abs/0904.4514.

[4] T. Cazenave, Semilinear Schrödinger equations, Courant Lecture Notes 10, Amer. Math. Soc. (2003). MR2002047 (2004j:35266)

[5] T. Chen, N. Pavlović, The quintic NLS as the mean field limit of a Boson gas with three-body interactions, J. Funct. Anal. 260 (4), 959-997 (2011). MR2747009

[6] T. Chen, N. Pavlović, On the Cauchy problem for focusing and defocusing Gross-Pitaevskii hierarchies, Discr. Contin. Dyn. Syst. 27 (2), 715-739, 2010. MR2600687 (2011f:35317)

[7] T. Chen, N. Pavlović, N. Tzirakis, Energy conservation and blowup of solutions for focusing GP hierarchies, Ann. Inst. H. Poincaré (C) Anal. Non-Lin. 27 (5), 1271-1290, 2010. MR2683760

[8] Z. Chen, C. Liu, On the Cauchy problem for Gross-Pitaevskii hierarchies, J. Math. Phys. 52, 032103 (2011). MR2814691 (2012d:35345)

[9] A. Elgart, L. Erdös, B. Schlein, H.-T. Yau, Gross-Pitaevskii equation as the mean field limit of weakly coupled bosons, Arch. Rat. Mech. Anal. 179, no. 2, 265-283 (2006). MR2209131 (2007b:81310)

[10] L. Erdös, B. Schlein, H.-T. Yau, Derivation of the Gross-Pitaevskii hierarchy for the dynamics of Bose-Einstein condensate, Comm. Pure Appl. Math. 59 (12), 1659-1741 (2006). MR:2257859(2007k:82070)

[11] L. Erdös, B. Schlein, H.-T. Yau, Derivation of the cubic non-linear Schrödinger equation from quantum dynamics of many-body systems, Invent. Math. 167 (2007), 515-614. MR2276262 (2007m:81258)

[12] L. Erdös, H.-T. Yau, Derivation of the nonlinear Schrödinger equation from a many body Coulomb system, Adv. Theor. Math. Phys. 5, no. 6, 1169-1205 (2001). MR1926667 (2004c:82075)

[13] J. Fröhlich, S. Graffi, S. Schwarz, Mean-field- and classical limit of many-body Schrödinger dynamics for bosons, Comm. Math. Phys. 271, no. 3, 681-697 (2007). MR2291792 $(2007 \mathrm{~m}: 82054)$

[14] J. Fröhlich, A. Knowles, A. Pizzo, Atomism and quantization, J. Phys. A 40, no. 12, 30333045 (2007). MR2313859 (2008c:82057)

[15] J. Fröhlich, A. Knowles, S. Schwarz, On the mean-field limit of bosons with Coulomb two-body interaction, Comm. Math. Phys. 288, no. 3, 1023-1059 (2009). MR2504864 (2010b:82039)

[16] M. Grillakis, M. Machedon, A. Margetis, Second-order corrections to mean field evolution for weakly interacting bosons. I, Comm. Math. Phys. 294 (1), 273-301 (2010). MR2575484 (2011j:81374)

[17] M. Grillakis, A. Margetis, A priori estimates for many-body Hamiltonian evolution of interacting boson system, J. Hyperbolic Differ. Equ. 5 (4), 857-883 (2008). MR2475483 (2010f:35321)

[18] K. Hepp, The classical limit for quantum mechanical correlation functions, Comm. Math. Phys. 35, 265-277 (1974). MR0332046 (48:10373)

[19] S. Klainerman, M. Machedon, On the uniqueness of solutions to the Gross-Pitaevskii hierarchy, Commun. Math. Phys. 279, no. 1, 169-185 (2008). MR.2377632 (2009a:35236)

[20] K. Kirkpatrick, B. Schlein, G. Staffilani, Derivation of the two-dimensional nonlinear Schrödinger equation from many body quantum dynamics, Amer. J. Math. 133 (2011), 91130. MR2752936

[21] E.H. Lieb, R. Seiringer, Proof of Bose-Einstein condensation for dilute trapped gases, Phys. Rev. Lett. 88, 170409 (2002).

[22] E.H. Lieb, R. Seiringer, J.P. Solovej, J. Yngvason, The mathematics of the Bose gas and its condensation, Birkhäuser (2005). MR2143817 (2006e:82001)

[23] E.H. Lieb, R. Seiringer, J. Yngvason, A rigorous derivation of the Gross-Pitaevskii energy functional for a two-dimensional Bose gas, Commun. Math. Phys. 224 (2001). MR.1868990 (2003a:82008) 
[24] I. Rodnianski, B. Schlein, Quantum fluctuations and rate of convergence towards mean field dynamics, Comm. Math. Phys. 291 (1), 31-61(2009). MR2530155(2011d:82059)

[25] B. Schlein, Derivation of Effective Evolution Equations from Microscopic Quantum Dynamics, Lecture notes for the minicourse held at the 2008 CMI Summer School in Zurich.

[26] H. Spohn, Kinetic Equations from Hamiltonian Dynamics, Rev. Mod. Phys. 52, no. 3, 569615 (1980). MR.578142(81e:82010)

[27] T. Tao, Nonlinear dispersive equations. Local and global analysis, CBMS 106, Amer. Math. Soc., Providence, RI, 2006. MR2233925 (2008i:35211)

Department of Mathematics, University of Texas at Austin, Austin, Texas 78712

E-mail address: tc@math.utexas.edu

Department of Mathematics, University of Texas at Austin, Austin, Texas 78712

E-mail address: natasa@math.utexas.edu 